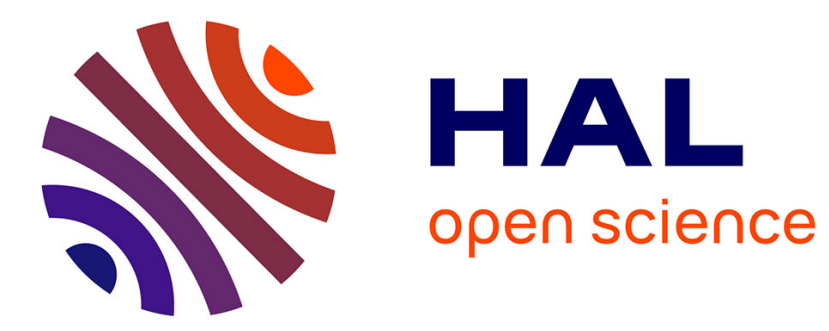

\title{
Spectroscope destiné à l'observation des radiations ultra-violettes
}

\author{
A. Cornu
}

\section{To cite this version:}

A. Cornu. Spectroscope destiné à l'observation des radiations ultra-violettes. J. Phys. Theor. Appl., 1879, 8 (1), pp.185-193. 10.1051/jphystap:018790080018500 . jpa-00237505

\section{HAL Id: jpa-00237505 https://hal.science/jpa-00237505}

Submitted on 1 Jan 1879

HAL is a multi-disciplinary open access archive for the deposit and dissemination of scientific research documents, whether they are published or not. The documents may come from teaching and research institutions in France or abroad, or from public or private research centers.
L'archive ouverte pluridisciplinaire HAL, est destinée au dépôt et à la diffusion de documents scientifiques de niveau recherche, publiés ou non, émanant des établissements d'enseignement et de recherche français ou étrangers, des laboratoires publics ou privés. 


\title{
SPEGTROSGOPE DESTINÉ A L'OBSERVATION DES RADIATIONS ULTRA-VIOLETTES ( $\left.{ }^{1}\right)$;
}

\author{
Par M. A. CORNU.
}

Le spectroscope que j'ai fait construire pour l'observation des radiations ultra-violettes ressemble complétement au spectroscope à deux prismes répandu dans les cabinets de Physique et les laboratoires de Chimie; il en diffère par la nature des matières transparentes qui entrent dans sa construction, car les matières employées ordinairement à la construction des spectroscopes, à savoir le crown-glass et le flint-glass, absorbent les radiations ultra-violettes au moins depuis la raie $\mathrm{O}$, c'est-à-dire depuis la longueur d'onde $\lambda=340$ millionièmes de millimètre.

La description des diverses parties de cet instrument me fournira l'occasion de résumer une série d'études relatives à l'observation des radiations très-réfrangibles.

Collimateur et lunette. - L'objectif qui forme la partie essentielle du collimateur comme de la lunette est sensiblement achromatique; il est composé d'une lentille biconvexe de quartz et d'une lentille divergente plan concave de spath d'Islande, toutes deux taillées perpendiculairement à l'axe optique. Cette composition de l'objectif est ex trèmement utile pour l'observation des spectres ultrayiolets, en général, et surtout du spectre solaire, parce que dans toute l'étendue des radiations ultra-violettes du Soleil, que l'atmosphère terrestre laisse arriver jusqu'à nous, les objectifs sont presque rigoureusement achromatiques. Il en résulte que la variation du tirage de la lunette et du collimateur nécessaire pour passer d'une partie à l'autre du spectre est sinon nulle, du moins extrêmement faible; l'observation photographique ou fluorescente des spectres devient alors extrêmement simple; dans le cas du spectre solaire, l'achromatisme est assez satisfaisant pour qu'on puisse obtenir sur le même cliché la totalité du spectre chimique, depuis la raie $\mathbf{F}$ jusqu'à la limite extrême (raies $\mathrm{S}, \mathrm{T}, \mathrm{U}$ ) la plus réfrangible.

La perfection des images n'est pas absolument la même sur tous

(1) Construit dans les ateliers de M. Jules Duboseq.

J. de Phys., t. VIIl. (Juin I 879.) 
les points du champ, parce que la surface focale est légèrement courbe; mais on peut, par une série d'essais méthodiques, arriver à déterminer un plan focal moyen qui rend l'ensemble de l'image très-satisfaisant. On peut encore améliorer les résultats par divers artifices qu'il serait trop long d'énumérer, et qui consistent soit à incliner légèrement le plan de la surface focale adoptée, soit à obtenir la compensation réciproque des petites aberrations d'obliquité et de courbure des objectifs et des prismes.

Pour donner une idée des conditions de l'achromatisme que

Fig. 1 .

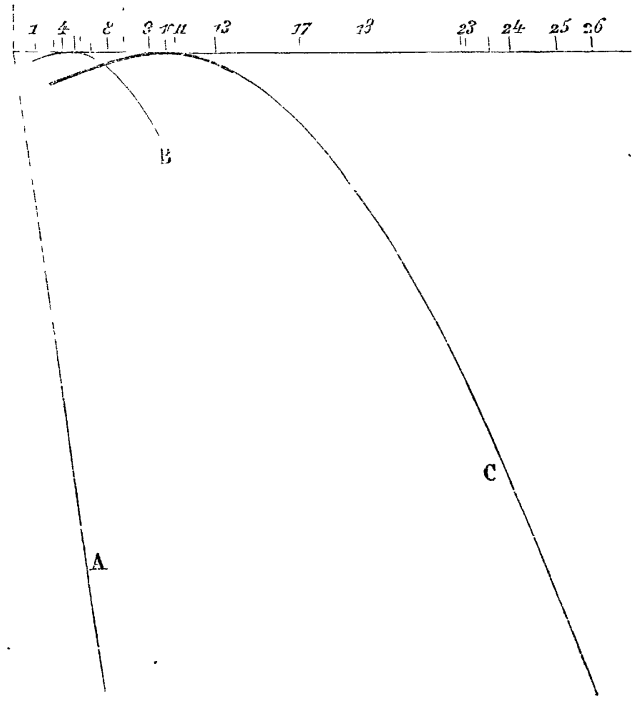

produit la combinaison quartz-spath d'Islande, je comparerai la variation de tirage de la lunette d'un spectroscope formé par des objectifs crown-glass (courbe B) dans l'observation du spectre solaire et du même spectroscope formé avec des objectifs quartz-spath (courbe $\mathrm{C}$ ) ayant sensiblement la même distance focale moyenne; enfin, j'indiquerai la variation focale correspondant à des objectifs simples de quartz (courbe A), employés d'ordinaire à ces observations ( fig. 1).

Les abscisses de ces courbes représentent les déviations minima du rayon ordinaire d'un prisme de $60^{\circ}$ en spath d'Islande, à l'échelle de $2^{\mathrm{mm}}, 5$ pour $\mathrm{I}^{\mathrm{o}}$. Les ordonnées représentent en vraie 
grandeur les variations du tirage de la lunette mise au point sur la radiation correspondant à l'abscisse, le tirage du collimateur restant fixe à une valeur moyenne correspondant à une distance focale d'environ $\mathrm{o}^{\mathrm{m}}, 45$. La ligne ainsi formée représente à peu près la courbe focale des diverses radiations dans une position moyenne du prisme, l'objectif étant fixe. D'après la figure, l'objectif serait situé au-dessus à la distance de $\mathrm{o}^{\mathrm{m}}, \mathbf{4 5}$; il en résulte que la partie du champ pour laquelle l'achromatisme est sensiblement parfait est celle qui se trouve dans le voisinage du minimum de distance focale. On aurait la loi de répartition linéaire ou de superposition des foyers des diverses radiations pour l'image d'un point lumineux en supposant que la courbe s'aplatisse de plus en plus par la diminution de l'échelle des abscisses.

On voit que l'objectif crown-glass-flint-glass, très-satisfaisant pratiquement au point de vue de l'achromatisme des images, n'est pas meilleur comme groupement des foyers que l'objectif quartzspath, pour une même étendue de spectre de part et d'autre du minimum de foyer ( 1 ).

Ces objectifs achromatiques ont donc un grand avantage, au point de vue de la facilité et de la rapidité des observations, sur les lentilles simples de quartz; mais ils ont un inconvénient qu'il est juste de signaler : c'est l'imparfaite transparence du spath d'Islande pour les radiations très-réfrangibles. Ces objectifs, en effet, laissent voir avec la plus grande facilité les raies de l'étincelle du cadmium jusqu'au no 25 (d'après la notation de M. Mascart, Annales de l'École Normale, ${ }^{\text {re }}$ série, t. IV, p. 7), raie 26 du même métal, les raies 27, 28, 29 du zinc, et même, à la rigueur, la raie $n^{\circ} 30$

(1) L'examen de ces courbes est très-instructif au point de vue de l'étude de l'achromatisme et donnerait lieu à une discussion dont voici brièvement les résultats :

$1^{\circ}$ Les courbes précédentes, qu'on peut obtenir enpiriquement par l'observation de la variation du tirage, se déduisent très-simplement de la courbe d'achromatisme (voir Journal de Physique, t. III, p. Io8) construite en prenant comme abscisse et comme ordonnée l'indice de réfraction de chaque radiation des deux matières à achromatiser.

$2^{\circ}$ Les petits changements de courbure ou d'écartement des deux lentilles ne modifient pas sensiblement les courbes représentées plus haut dans leur forme absolue; la modification équivaut à un changement dans la direction des axes coordonnés, de sorte que l'on peut se rendre immédiatement un compte très-exact de la répartitiun des foyers si la distance focale minimum correspond à une autre radiation. 
de l'aluminium (notation de M. Soret, Archives de la bibliothèque de Genève, mars 1878 , t. LXI) ; mais les raies 31 et 32 de l'aluminium sont complétement absorbées : avec les lentilles simples de quartz (et un prisme de quartz), ces raies, les plus réfrangibles qu'on ait observées jusqu'ici, apparaissent, au contraire, avec un assez vif éclat.

On atténue le pouvoir absorbant de ces objectifs en diminuant autant que possible l'épaisseur de la lentille divergente de spath d'Islande.

Les rayons de courbures adoptés pour la taille des objectifs sont les suivants; ils sont exprimés en fonction de la distance focale $\mathbf{F}$ de la lumière jaune de la soude (raie $\mathbf{D})$ :

\section{Quartz perpendiculaire à l'axe.}

$$
\begin{array}{rrr}
\text { Surface convexe extérieure...... } & \mathbf{R}=-0,4 \text { I5 I } \mathbf{F} \\
\text { intérieure.... } & \mathbf{R}^{\prime}=0,363 \text { I } \mathbf{F}
\end{array}
$$

Spath d'Islande perpendiculaire à l'axe.

$$
\begin{aligned}
\text { Surface concave intérieure ..... } & \mathbf{R}^{\prime}=-0,363_{\text {I }} \mathbf{F} \\
\text { extérieure ..... } & \mathbf{R}^{\prime \prime}=\infty
\end{aligned}
$$

Ces courbures conviennent plus spécialement à l'étude du spectre solaire ultra-violet, qui s'étend jusqu'à la longueur d'onde $\lambda=294$, c'est-à-dire presque jusqu'à la raie $\mathrm{n}^{\circ} 17 \mathrm{du}$ cadmium. Dans cette étendue, le champ est symétrique par rapport à la raie $n^{0} 10$, c'est-à-dire de longueur d'onde $\lambda=347$, et présente une très-faible courbure. Si l'on diminuait un peu le rayon de la courbure $R$ de la surface extérieure du quartz, on repousserait vers la partie la plus réfrangible le sommet de la courbe, c'est-à-dire le centre de symétrie du champ.

Ces courbures ne sont pas celles qui donnent théoriquement le minimum d'aberration de sphéricité; mais au point de vue pratique ce sont celles qui donnent les meilleures images, à cause des difficultés que présente la taille et le poli du spath d'Islande; en effet, les opticiens sont mieux outillés pour polir des surfaces planes que les surfaces courbes de spath; on a donc intérêt à supprimer la face très-peu courbe donnée par la théorie et à la 
remplacer par une face plane, qui, d'ailleurs, altère fort peu les conditions du minimum d'aberration sphérique.

On a essayé, pour atténuer l'imperfection de la surface concave de spath d'Islande, de coller avec un mastic réfringent (le baume de Canada ou le mastic en larmes) les deux surfaces en contact; mais le pouvoir absorbant de ces résines est tellement considérable pour les radiations ultra-violettes que, malgré la minceur de la couche interposée $\left(\frac{1}{10}\right.$ de millimètre à peine) et la transparence de la matière employée, l'absorption commençait déjà à la raie $n^{0} 10$ du cadmium $(\lambda=346)$, c'est-à-dire avant la fin du spectre solaire. Il a suffi de plonger les objectifs dans l'éther pour dissoudre le mastic et rendre aux objectifs leur transparence primitive.

Certains liquides peuvent néanmoins être utilisés pour le collage. Ainsi l'eau, l'alcool ou mieux la glycérine bien pure et bien décantée ne produisent aucune absorption sensible jusqu'à l'extrémité du spectre observable, sous l'épaisseur nécessaire à cette opération. On pourrait donc, dans certains cas où la finesse des images devrait être poussée un peu plus loin, se servir de cet artifice très-simple, à la condition de ne pas prolonger l'action des liquides sur le spath, dont la surface polie pourrait être altérée.

Enfin, une précaution qui contribue à la perfection des images consiste à adopter pour les lentilles de quartz des épaisseurs égales et de pouvoir rotatoire inverse; on élimine ainsi, par compensation, des effets secondaires qui ne pourraient que troubler la netteté des foyers.

Prismes. - Le choix de la nature des prismes a nécessité aussi diverses études. Le spath d'Islande a l'avantage de présenter une très-forte dispersion dans le rayon ordinaire, mais il a l'inconvénient d'être difficile à bien tailler, et surtout d'absorber les radiations très-réfrangibles. Ce dernier inconvénient est bien plus grave pour le prisme, dont l'épaisseur moyenne est nécessairement considérable, que pour les objectifs dont l'épaisseur peut être beaucoup réduite. Aussi, avec un prisme de spath d'Islande, est-il difficile de dépasser la raie $n^{\circ} 26$ du cadmium; encore faut-il observer le rayon extraordinaire qui paraît un peu moins absorbé que le rayon ordinaire.

La taille la plus favorable du prisme de spath d'Islande est celle pour laquelle l'arête est parallèle à l'axe optique; l'angle le plus 
usité est celui de $60^{\circ}$. Les deux spectres ordinaire et extraordinaire sont entic̀rement séparés et présentent des dispersions à peu près dans le rapport de 2 à $\mathbf{I}$.

Le quartz doit être préféré au spath si l'on veut observer jusqu'à l'extrême limite des radiations réfrangibles; d'un autre côté, il prend un poli d'une perfection incomparable à cause de sa grande dureté. Il offre toutefois, dans ses propriétés optiques, diverses particularités gênantes dont il a fallu éliminer les effets avant d'obtenir les meilleurs résultats.

La première particularité défavorable est la faiblesse de la biréfringence; les deux spectres ordinaire et extraordinaire sont superposés. On a souvent tourné cette difficulté en profitant de la polarisation des deux rayons pour éteindre l'un d'eux par un polariseur ou un analyseur convenablement placé ; mais on tombe dans des difficultés d'un autre ordre provenant, d'une part, du pouvoir rotatoire des lentilles de quartz qui constituent les objectifs du spectroscope, et, d'autre part, de la construction d'un polariseur n'absorbant pas les radiations ultra-violeltes.

Quoique je sois parvenu à une disposition additionnelle assez simple pour résoudre la difficulté, je n'ai pas considéré cette solution comme applicable à un spectroscope d'usage courant. En effet, la multiplicité des surfaces réfringentes élimine une quantité notable de lumière, et, comme on opère déjà sur des rayons dont l'intensité est diminuée de moitié par la double réfraction, on risque de réduire à une fraction trop faible l'éclat des radiations à observer.

Il faut donc éviter la biréfringence et, pour cela, prendre l'axe optique du quartz perpendiculaire au plan bissecteur du prisme. Mais ici se présente la seconde particularité défavorable du quartz, à savoir le pouroir rotatoire, qui dédouble les rayons incidents en deux rayons circulaires de rotation inverse. La biréfringence est, il est vrai, très-faible et n'a pas d'inconvénient sensible dans certaines expériences, comme celles de M. Stockes, lorsque l'on opère par projection sur un écran fluorescent; mais, dans les observations délicates, les images sont dédoublées et l'écartement croît rapidement avec la réfrangibilité. Ainsi on retombe sur les mêmes inconvénients.

Mais cette fois on peut aisément tourner la difficulté : il suffit 
de composer le prisme de deux moitiés de rotation contraire, la face intérieure étant perpendiculaire à l'axe. On obtient ainsi des images dont la netteté ne dépend plus que de la perfection des faces.

Par l'artifice indiqué plus haut, à savoir le collage des deux surfaces contiguës à l'aide d'une goutte d'eau, d'alcool ou de glycérine, on élimine l'influence de ces deux surfaces, tant au point de vue de l'imperfection de leur forme qu'à celui de la perte de lumière causée par l'interposition d'une lame d'air.

Dans le cas où l'on veut obtenir une dispersion double, la compensation du pouvoir rotatoire est plus simple encore: il suffit d'employer deux prismes de quartz ayant leurs plans bissecteurs perpendiculaires à l'axe optique, mais présentant des pouvoirs rotatoires de sens contraires. La dispersion est alors sensiblement la même que celle du rayon ordinaire du spath d'Islande, et on a l'avantage de la transparence complète et de la perfection du poli des faces.

C'était la disposition adoptée dans le spectroscope que j'ai eu l'honneur de mettre sous les yeux de la Société de Physique.

Mode d'observation. - La précision des observations atteint un haut degré de perfection lorsqu'on emploie la Photographie. Les dispositions très-simples à adopter ont été décrites par différents observateurs ( $\left.{ }^{1}\right)$. Les clichés que l'on obtient ainsi ont une grande finesse et supportent des grossissements de 25 diamètres. On peut les conserver et les étudier à loisir, ce qui rend les observations encore plus précieuses. Le petit châssis porte-plaque que j’ai décrit $\left({ }^{2}\right)$ s'applique au spectroscope avec la plus grande facilité.

Mais, pour les usages courants et même pour préparer l'emploi de la Photographie, le mode d'observation le plus commode est l'emploi d'un oculaire fluorescent imaginé par M. Baille ( $\left.{ }^{3}\right)$, et amené à une grande perfection par M. Soret (voir Journal de Physique, t. III, p. 253). Al'aide de cet oculaire ( $f g .2$ ), formé essentiellement d'une lame fluorescente $f f$ (verre d'urane) de $\mathrm{r}^{\mathrm{mm}}$ d'épaisseur, que l'on observe obliquement par transparence dans le plan des

(') Becquerel, La lumière. - Miscart, Annales de l'École Normale, I ${ }^{\mathrm{re}}$ série, t. I.

- Corxu, ibid., $2^{\circ}$ série, t. III, p. $24 \mathrm{I}$.

(') Ibid.

( $\left.{ }^{3}\right)$ Annales du Conservatoire des Arts et Métiers, 1867. 
raies sous une incidence de $20^{\circ}$ à $30^{\circ}$, les raies brillantes des vapeurs métalliques s'observent avec une grande facilité : il suffit de faire jaillir l'étincelle d'une bobine d'induction de grandeur moyenne, avec intercalation d'une bonne bouteille de Leyde entre deux électrodes du métal à étudier. La fente peut être amenée jusqu'à une assez grande finesse, à la condition de mettre la lame de verre d'urane exactement au foyer des radiations observées.

Pour s'exercer à ces observations, je conseille d'étudier d'abord Fig. 2.

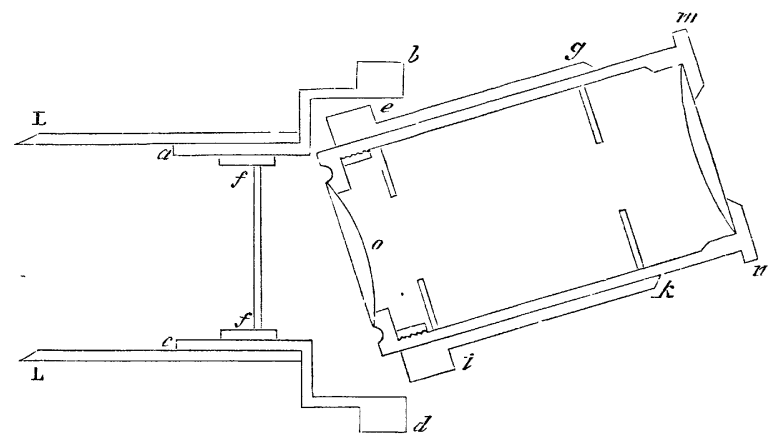

le spectre du magnésium, qui présente une raie quadruple excessivement intense $(\lambda=280)$ un peu au delà de la limite du spectre solaire. C'est, toutes choses égales d'ailleurs, la radiation ultraviolette très-réfrangible de beaucoup la plus intense quel'on puisse obtenir avec les métaux usuels. Les quatre composantes sont assez resserrées pour que l'ensemble puisse servir dans une foule de cas de source monochromatique.

Ensuite on s'exercera à observer les raies du cadmium, puis celles du zinc et enfin celles de l'aluminium, dont les plus réfrangibles forment la limite des radiations observables.

L'étude du spectre solaire est plus délicate lorsqu'on veut la pousser un peu loin. Il faut prendre beaucoup de précautions pour éviter les reflets à l'intérieur de l'oculaire fluorescent, et pour cela ajouter, à $\mathrm{o}^{\mathrm{m}}, 02$ en avant de la lame fluorescente, un diaphragme d'ouverture aussi petite que possible; malgré cette précaution, on n'atteint pas la limite à laquelle on parvient avec la Photographie.

Pour obtenir plus de finesse dans les détails, sauf à perdre un peu d'éclat, il est bon de réduire l'épaisseur du verre d'urane à 
J. MACE DE LÉPINAY. - SIPHON ENREGISTREUR. 193 $\mathrm{o}^{\mathrm{m}}, \tilde{\jmath}$; on doit même le remplacer, ainsi que l'indique M. Soret, par une petite cuve d'une solution d'esculine pour observer la région LMN, dans laquelle la fluorescence du verre d'urane est très-faible.

Repères. - La position des raies s'obtient surtout par comparaison avec un spectre déjà connu, et en particulier avec les raies du spectre solaire. On projette la raie dont on veut connaître la longueur d'onde sur le trait-réticule du verre d'urane, et l'on substitue à la source un faisceau solaire; mais on ne peut guère dépasser la longueur d'onde $\lambda=300$.

Si la lunette du spectroscope est munie d'un mouvement micrométrique, on peut opérer sur toute l'étendue du spectre ultraviolet en rapportant les observations aux raies du cadmium, du zinc et de l'aluminium, qui forment une échelle suivant les conventions de MM. Mascart, Soret et Sarrazin.

Pour des observations moins précises, on peut se contenter de l'image d'une échelle diviséc réfléchie sur la deuxième face du dernier prisme, comme dans les spectroscopes ordinaires. L'image de cette échelle est amenée dans le plan de la lame fluorescente, à la hauteur de la croisée des traits-réticules. On amène la raie à définir (observée obliquement par fluorescence) sur la croisée des traits, puis on abaisse l'oculaire de manière à voir par transparence les traits de l'échelle divisée: la croisée des traits définit sur l'échelle la position de la raie. On peut ainsi, indirectement il est vrai, mais d'une manière simple ct rapide, construire la carte des spectres ultra-violets avec presque autant de facilité que celle des spectres visibles. 